\title{
MULTIVALUED ATTRACTORS AND THEIR APPROXIMATION: APPLICATIONS TO THE NAVIER-STOKES EQUATIONS
}

\author{
MICHELE COTI ZELATI, FLORENTINA TONE
}

\begin{abstract}
This article is devoted to the study of multivalued semigroups and their asymptotic behavior, with particular attention to iterations of set-valued mappings. After developing a general abstract framework, we present an application to a time discretization of the two-dimensional Navier-Stokes equations. More precisely, we prove that the fully implicit Euler scheme generates a family of discrete multivalued dynamical systems, whose global attractors converge to the global attractor of the continuous system as the time-step parameter approaches zero.
\end{abstract}

\section{INTRODUCTION}

The variety of questions related to evolution equations arising from fluid mechanics problems constitutes a challenging and fascinating area of mathematics, which has attracted the attention of a wide number of researchers for many years. One important aspect, among others, is the understanding of the behavior of solutions to differential equations as time goes to infinity. For autonomous systems, this translates into the study of the properties of a semigroup of operators $\{S(t)\}_{t \geq 0}$, also called a dynamical system, acting on a phase space $X$, typically a Banach space or, more generally, a complete metric space [4, 7, 9, 23]. Notice that the parameter $t$ could be regarded as discrete, if dealing with a difference equation, or as continuous, in the case of a differential equation.

When global existence and uniqueness of solutions can be proved, dynamical systems arise as solution operators assigning to a certain initial condition $x \in X$ the corresponding solution $x(t)=S(t) x$ of the evolution problem under consideration. Unfortunately, in many instances, uniqueness of solutions may be hard to prove, or even out of reach. In this case, one has to deal with a so-called multivalued semigroup of operators, for which $S(t) x$ is the set of all possible solutions at time $t$. Multivalued semigroups have been investigated by many authors, and are particularly powerful in the study of abstract differential inclusions, doubly nonlinear equations, gradient flows and stochastic partial differential equations [2, 3, 5, 14, 17, 18].

From the large time behavior viewpoint, the most relevant object is the so-called global attractor, namely, the unique compact subset of the phase space which is at the same time invariant and attracting. As noted in [27], it is of crucial importance to understand whether the longterm dynamics of a system possessing global attractor can be properly approximated by discrete attractors of discrete dynamical systems generated, for example,

Date: November 6, 2018.

2000 Mathematics Subject Classification. 37L05, 37L15, 65P99, 35Q35.

Key words and phrases. Multivalued dynamical systems, global attractors, time discretization, NavierStokes equations, implicit Euler scheme. 
by numerical schemes associated to the evolution problem under concern. This issue has been widely investigated and the interested reader is referred to, e.g., [10, 11, 12, 13, 19, 21, 26].

1.1. The physical model and its approximation. Let $\Omega \subset \mathbb{R}^{2}$ be a bounded domain with smooth boundary $\partial \Omega$. For $t \geq 0$ and $\nu>0$, we consider the two-dimensional Navier-Stokes equations [20, 22]

$$
\left\{\begin{array}{l}
\partial_{t} u-\nu \Delta u+(u \cdot \nabla) u+\nabla p=f, \\
\operatorname{div} u=0
\end{array}\right.
$$

where $f$ is an autonomous incompressible forcing term. The system is supplemented with the nonslip boundary condition

$$
\left.u(x, t)\right|_{x \in \partial \Omega}=0
$$

and the initial condition

$$
u(x, 0)=u_{0}(x)
$$

along with its time discretization provided by the fully implicit Euler scheme [24]

$$
\frac{u^{n}-u^{n-1}}{k}-\nu \Delta u^{n}+\left(u^{n} \cdot \nabla\right) u^{n}+\nabla p^{n}=f, \quad u^{0}=u_{0}
$$

Due to its nonlinear nature, uniqueness of solution to such a numerical approximation can be proved only by restricting the time-step parameter $k>0$ to be small enough. To be more precise, such a restriction depends on the initial datum $u_{0}$ and does not therefore allow to define a single-valued discrete semigroup of operators in the classical sense. Nonetheless, we will be able to show that, in fact, this difficulty can be overcome by defining a family of multivalued discrete semigroups $\left\{S_{k}, 0<k \leq \kappa_{1}\right\}$, where $\kappa_{1}>0$ is constant and independent of $u_{0}$.

Taking advantage of previous results contained in [25], we will address the issue of the existence of a family of discrete global attractors $\mathcal{A}_{k}$ of $S_{k}$ and we will prove that $\mathcal{A}_{k} \rightarrow \mathcal{A}$ as $k \rightarrow 0$ in a suitable sense, where $\mathcal{A}$ is the global attractor of the single-valued dynamical system generated by (1.1) $-(\underline{1.3})$.

1.2. Structure of the paper. In the next section we develop, along the lines of [14, the abstract machinery of multivalued dynamical systems needed to prove the results described above. In particular, we will focus on the existence of multivalued global attractors, and prove a convergence theorem for families of discrete attractors depending on a parameter. Section 3 is dedicated to the applications of the abstract theory to the Navier-Stokes equations and their approximation. It is shown that the fully implicit Euler scheme generates a multivalued discrete dynamical system whose asymptotic dynamics is properly related to the one of the Navier-Stokes equations. 


\section{The ABstract Framework}

We here develop in an abstract way the main tools needed for the study of multivalued semigroups and their asymptotic behavior. Some results are more or less already known thanks to the works [2] and [14]. We also refer to [6] and to the more recent work [8] for a fairly complete overview on the subject. Except for the approximation results, equivalent statements of the theorems in this section can be found in [14]. We report here alternative proofs for completeness and review purposes.

While the above papers mainly dealt with continuous-time semigroup, we are more interested in discrete dynamical systems generated by numerical schemes for which uniqueness of solutions may not hold. In particular, we will address the question of under what conditions a discrete multivalued semigroup can approximate the longtime behavior of a continuous one.

2.1. Multivalued Semigroups. Let $(X,\|\cdot\|)$ be a real Banach space, and let $\mathbb{T}$ be either $\mathbb{R}^{+}=[0, \infty)$ or $\mathbb{N}$. A one-parameter family of set-valued maps $S(t): 2^{X} \rightarrow 2^{X}$ is a multivalued semigroup (m-semigroup) if it satisfies the following properties:

(S.1) $S(0)$ is the identity on $2^{X}$;

(S.2) $S(t+\tau)=S(t) S(\tau)$, for all $t, \tau \in \mathbb{T}$.

To simplify the notation, if $x \in X$ we will write $S(t) x$ in place of $S(t)\{x\}$ and, as customary, for any set $\mathcal{B} \in 2^{X}$, we will assume

$$
S(t) \mathcal{B}=\bigcup_{x \in \mathcal{B}} S(t) x .
$$

The m-semigroup is said to be closed if it fulfills the further property:

(S.3) $S(t)$ is a closed map for every $t \in \mathbb{T}$, meaning that if $x_{n} \rightarrow x$ and $y_{n} \in S(t) x_{n}$ is such that $y_{n} \rightarrow y$, then $y \in S(t) x$.

Remark 2.1. The notion of continuity of a set-valued map is not as immediate as in the single-valued case. An m-semigroup is called upper semicontinuous if given $x \in X$ and a neighborhood $\mathcal{U}(S(t) x)$ of $S(t) x$, there exists $\delta>0$ such that

$$
\|x-y\|<\delta \quad \Rightarrow \quad S(t) y \subset \mathcal{U}(S(t) x) .
$$

On the other hand, $S(t)$ is defined to be lower semicontinuous if given $x_{n} \rightarrow x$ and $y \in S(t) x$, there exists $y_{n} \in S(t) x_{n}$ such that $y_{n} \rightarrow y$. Finally, $S(t)$ is continuous if it is at the same time lower and upper semicontinuous. In general, it is not true that a continuous m-semigroup is closed (as it is in the single-valued case), the problem being that $S(t) x$ might not be a closed set (if $S(t) x$ is closed for any $x \in X$, the $S(t)$ is said to have closed values). Nonetheless, if $S(t)$ is upper semicontinuous and has closed values, then $S(t)$ is closed (see [1]).

The positive orbit of $\mathcal{B}$, starting at $t \in \mathbb{T}$, is the set

$$
\gamma_{t}(\mathcal{B})=\bigcup_{\tau \geq t} S(\tau) \mathcal{B}
$$

where we agree to set $\gamma(\mathcal{B})=\gamma_{0}(\mathcal{B})$. A function $y: \mathbb{T} \rightarrow X$ is said to be a trajectory starting at $y_{0} \in X$ if $y(0)=y_{0}$ and $y(t+\tau) \in S(t) y(\tau)$ for every $t, \tau \in \mathbb{T}$. 
Limit Sets. For any $\mathcal{B} \in 2^{X}$, the set

$$
\omega(\mathcal{B})=\bigcap_{t \in \mathbb{T}} \overline{\gamma_{t}(\mathcal{B})}
$$

is called the $\omega$-limit set of $\mathcal{B}$. The following characterization of $\omega(\mathcal{B})$ holds true in the multivalued case, and the proof is identical to the single-valued counterpart.

Lemma 2.2. A point $x \in X$ belongs to $\omega(\mathcal{B})$ if and only if there are sequences $t_{n} \rightarrow \infty$ and $x_{n} \in S\left(t_{n}\right) \mathcal{B}$ such that $x_{n} \rightarrow x$ as $n \rightarrow \infty$.

A nonempty set $\mathcal{B} \in 2^{X}$ is invariant for $S(t)$ if

$$
S(t) \mathcal{B}=\mathcal{B}, \quad \forall t \in \mathbb{T} .
$$

If $S(t) \mathcal{B} \subset \mathcal{B}$, then $\mathcal{B}$ is said to be positively invariant.

Dissipativity. A set $\mathcal{B}_{0} \in 2^{X}$ is an absorbing set for the m-semigroup $S(t)$ if for every bounded set $\mathcal{B} \in 2^{X}$ there exists $t_{\mathcal{B}} \in \mathbb{T}$ such that

$$
S(t) \mathcal{B} \subset \mathcal{B}_{0}, \quad \forall t \geq t_{\mathcal{B}}
$$

Given two nonempty sets $\mathcal{B}, \mathcal{C} \in 2^{X}$, the Hausdorff semidistance between $\mathcal{B}$ and $\mathcal{C}$ (in $X$ ) is defined as

$$
\operatorname{dist}(\mathcal{B}, \mathcal{C})=\sup _{b \in \mathcal{B}} \inf _{c \in \mathcal{C}}\|b-c\|
$$

Notice that, in general, $\operatorname{dist}(\mathcal{B}, \mathcal{C}) \neq \operatorname{dist}(\mathcal{C}, \mathcal{B})$. A nonempty set $\mathcal{C} \in 2^{X}$ is attracting if for every bounded set $\mathcal{B}$ we have

$$
\lim _{t \rightarrow \infty} \operatorname{dist}(S(t) \mathcal{B}, \mathcal{C})=0 .
$$

The m-semigroup $S(t)$ is called dissipative if it possesses a bounded absorbing set. Some authors prefer to require compactness (instead of only boundedness) in the notion of dissipativity, a strategy which has been successful expecially in parabolic problems [16].

The Global Attractor. A nonempty compact set $\mathcal{A} \in 2^{X}$ is said to be the global attractor of $S(t)$ if

(A.1) $\mathcal{A}$ is invariant;

(A.2) $\mathcal{A}$ is an attracting set.

Remark 2.3. The global attractor, if it exists, is necessarily unique. Moreover, it enjoys the following maximality and minimality properties:

(i) let $\tilde{\mathcal{A}}$ be a bounded set satisfying (A.1), Then $\mathcal{A} \supset \tilde{\mathcal{A}}$;

(ii) let $\tilde{\mathcal{A}}$ be a closed set satisfying $(\mathrm{A} .2)$, Then $\mathcal{A} \subset \tilde{\mathcal{A}}$.

In order to state the result on the existence of the global attractor, we need a definition. Given a bounded set $\mathcal{B} \in 2^{X}$, the Kuratowski measure of noncompacteness $\alpha(\mathcal{B})$ of $\mathcal{B}$ is defined as

$$
\alpha(\mathcal{B})=\inf \{\delta: \mathcal{B} \text { has a finite cover by balls of } X \text { of diameter less than } \delta\} .
$$

We list hereafter some properties of $\alpha$.

$(\mathrm{K} .1) \alpha(\mathcal{B})=\alpha(\overline{\mathcal{B}})$; 
(K.2) $\mathcal{B}_{1} \subset \mathcal{B}_{2}$ implies that $\alpha\left(\mathcal{B}_{1}\right) \leq \alpha\left(\mathcal{B}_{2}\right)$;

(K.3) $\alpha(\mathcal{B})=0$ if and only if $\overline{\mathcal{B}}$ is compact;

(K.4) if $\left\{\mathcal{B}_{t}\right\}_{t \in \mathbb{T}}$ is a family of nonempty closed sets such that $\mathcal{B}_{t_{2}} \subset \mathcal{B}_{t_{1}}$ for $t_{2}>t_{1}$ and $\lim _{t \rightarrow \infty} \alpha\left(\mathcal{B}_{t}\right)=0$, then $\mathcal{B}=\bigcap_{t \in \mathbb{T}} \mathcal{B}_{t}$ is nonempty and compact;

(K.5) if $\left\{\mathcal{B}_{t}\right\}_{t \in \mathbb{T}}$ and $\mathcal{B}$ are as above, given any $t_{n} \rightarrow \infty$ and any $x_{n} \in \mathcal{B}_{t_{n}}$, there exist $x \in \mathcal{B}$ and a subsequence $x_{n_{k}} \rightarrow x$.

Theorem 2.4. Suppose that the closed m-semigroup $S(t)$ possesses a bounded absorbing set $\mathcal{B}_{0} \in 2^{X}$ and

$$
\lim _{t \rightarrow \infty} \alpha\left(S(t) \mathcal{B}_{0}\right)=0
$$

Then $\omega\left(\mathcal{B}_{0}\right)$ is the global attractor of $S(t)$.

Proof. First, we prove that $\omega\left(\mathcal{B}_{0}\right)$ is nonempty and compact. Let $t_{0} \in \mathbb{T}$ be such that

$$
S(\tau) \mathcal{B}_{0} \subset \mathcal{B}_{0}, \quad \forall \tau \geq t_{0} .
$$

If $t \geq t_{0}$, we have

$$
\begin{aligned}
\gamma_{t}\left(\mathcal{B}_{0}\right) & =\bigcup_{\tau \geq t} S(\tau) \mathcal{B}_{0}=\bigcup_{\tau \in \mathbb{T}} S(\tau+t) \mathcal{B}_{0} \\
& =\bigcup_{\tau \in \mathbb{T}} S\left(t-t_{0}\right) S\left(\tau+t_{0}\right) \mathcal{B}_{0} \subset \bigcup_{\tau \in \mathbb{T}} S\left(t-t_{0}\right) \mathcal{B}_{0}=S\left(t-t_{0}\right) \mathcal{B}_{0} .
\end{aligned}
$$

Therefore, in light of (K.1), (K.2) and (2.1),

$$
\lim _{t \rightarrow \infty} \alpha\left(\overline{\gamma_{t}\left(\mathcal{B}_{0}\right)}\right)=\lim _{t \rightarrow \infty} \alpha\left(\gamma_{t}\left(\mathcal{B}_{0}\right)\right)=0 .
$$

Since the sets $\gamma_{t}\left(\mathcal{B}_{0}\right)$ are nested, from (K.4) we conclude that

$$
\omega\left(\mathcal{B}_{0}\right)=\bigcap_{t \in \mathbb{T}} \overline{\gamma_{t}\left(\mathcal{B}_{0}\right)}
$$

is nonempty and compact.

To prove $\omega\left(\mathcal{B}_{0}\right)$ is attracting, argue by contradiction, and assume that there exist $\varepsilon>0$ and sequences $t_{n} \rightarrow \infty$ and $y_{n} \in S\left(t_{n}\right) \mathcal{B}_{0}$ such that

$$
\inf _{y \in \omega\left(\mathcal{B}_{0}\right)}\left\|y_{n}-y\right\| \geq \varepsilon .
$$

Since $y_{n} \in S\left(t_{n}\right) \mathcal{B}_{0}$, it follows that $y_{n} \in \gamma_{t_{n}}\left(\mathcal{B}_{0}\right)$. By properties (K.4) and (K.5), there exist $z \in \omega\left(\mathcal{B}_{0}\right)$ and a subsequence $y_{n_{k}} \in S\left(t_{n_{k}}\right) \mathcal{B}_{0}$ such that $y_{n_{k}} \rightarrow z$, which is a contradiction.

It remains to show that $\omega\left(\mathcal{B}_{0}\right)$ is invariant. Let $t_{n} \rightarrow \infty$ and $y_{n} \in S\left(t_{n}\right) \mathcal{B}_{0}$ be any sequences. We claim that there exists $y \in \omega\left(\mathcal{B}_{0}\right)$ such that $y_{n} \rightarrow y$ up to a subsequence. Since $\omega\left(\mathcal{B}_{0}\right)$ is attracting, we know that

$$
\lim _{n \rightarrow \infty} \operatorname{dist}\left(S\left(t_{n}\right) \mathcal{B}_{0}, \omega\left(\mathcal{B}_{0}\right)\right)=0 .
$$

As a consequence,

$$
\lim _{n \rightarrow \infty} \inf _{z \in \omega\left(\mathcal{B}_{0}\right)}\left\|y_{n}-z\right\|=0
$$

from which we deduce the existence of a sequence $z_{n} \in \omega\left(\mathcal{B}_{0}\right)$ such that

$$
\lim _{n \rightarrow \infty}\left\|y_{n}-z_{n}\right\|=0 \text {. }
$$


From the compactness of $\omega\left(\mathcal{B}_{0}\right)$ we readily get $y \in \omega\left(\mathcal{B}_{0}\right)$ and a subsequence $n_{k}$ such that $z_{n_{k}} \rightarrow y$, and, in turn, $y_{n_{k}} \rightarrow y$.

Let $x \in \omega\left(\mathcal{B}_{0}\right)$ and $t \in \mathbb{T}$. By Lemma 2.2, there exist sequences $t_{n} \rightarrow \infty$ and $y_{n} \in$ $S\left(t_{n}\right) \mathcal{B}_{0}$ such that $y_{n} \rightarrow x$ as $n \rightarrow \infty$. Since

$$
S\left(t_{n}\right) \mathcal{B}_{0}=S(t) S\left(t_{n}-t\right) \mathcal{B}_{0}
$$

we obtain that $y_{n} \in S(t) z_{n}$, with $z_{n} \in S\left(t_{n}-t\right) \mathcal{B}_{0}$. By the above claim, there exists $y \in \omega\left(\mathcal{B}_{0}\right)$ such that $z_{n} \rightarrow y$ up to a subsequence. The fact that $S(t)$ is closed then yields the following implication

$$
z_{n} \rightarrow y, \quad S(t) z_{n} \ni y_{n} \rightarrow x \quad \Rightarrow \quad x \in S(t) y,
$$

namely the inclusion $\omega\left(\mathcal{B}_{0}\right) \subset S(t) \omega\left(\mathcal{B}_{0}\right)$. Turning to the opposite one, we now know that

$$
S(t) \omega\left(\mathcal{B}_{0}\right) \subset S(\tau) S(t) \omega\left(\mathcal{B}_{0}\right)=S(t+\tau) \omega\left(\mathcal{B}_{0}\right)
$$

for every $t, \tau \in \mathbb{T}$. Since $\omega\left(\mathcal{B}_{0}\right)$ is attracting, for every neighborhood $\mathcal{U}\left(\omega\left(\mathcal{B}_{0}\right)\right)$ of $\omega\left(\mathcal{B}_{0}\right)$, there exists $t_{\mathcal{U}} \in \mathbb{T}$ such that

$$
S(t+\tau) \omega\left(\mathcal{B}_{0}\right) \subset \mathcal{U}\left(\omega\left(\mathcal{B}_{0}\right)\right), \quad \forall \tau \geq t_{\mathcal{U}}
$$

Thus $S(t) \omega\left(\mathcal{B}_{0}\right) \subset \mathcal{U}\left(\omega\left(\mathcal{B}_{0}\right)\right)$, where $\mathcal{U}\left(\omega\left(\mathcal{B}_{0}\right)\right)$ is arbitrary, so that

$$
S(t) \omega\left(\mathcal{B}_{0}\right) \subset \overline{\omega\left(\mathcal{B}_{0}\right)}=\omega\left(\mathcal{B}_{0}\right), \quad \forall t \in \mathbb{T},
$$

which concludes the proof.

2.2. Discrete Approximation Of Multivalued Semigroups. We now turn our attention to the approximation of continuous-time m-semigroups by discrete ones, exploring how certain properties that hold true in the discrete regime are carried over to the continuous limit.

Remark 2.5. Given a set-valued map $S: 2^{X} \rightarrow 2^{X}$, we can define a discrete m-semigroup by

$$
S(n)=S^{n}, \quad \forall n \in \mathbb{N} .
$$

Properties (S.1) and (S.2) are trivially satisfied, and, in this case, we will write that $\{S\}_{n \in \mathbb{N}}$ (instead of the redundant $\left\{S^{n}\right\}_{n \in \mathbb{N}}$ ) is a discrete m-semigroup.

Turning to our approximation problem, let $\{S(t)\}_{t \in \mathbb{R}^{+}}$be a closed m-semigroup, $\kappa_{0}$ a positive constant, and consider a family of discrete closed m-semigroups $\left\{S_{k}, 0<k \leq\right.$ $\left.\kappa_{0}\right\}_{n \in \mathbb{N}}$ where, for each fixed $k$, the map $S_{k}: 2^{X} \rightarrow 2^{X}$ satisfies the usual semigroup properties

$$
S_{k}^{0}=\operatorname{Id}_{2^{x}}, \quad S_{k}^{n+m}=S_{k}^{n} S_{k}^{m}, \quad \forall n, m \in \mathbb{N} .
$$

Such maps arise in the study of numerical schemes associated to evolutionary equations, in which either the discretization problem or the differential system (or both) might not enjoy any uniqueness property of solutions. From the point of view of the longtime behavior of solutions, it is therefore interesting to understand under what conditions the asymptotic features of the continuous-time m-semigroup can be properly approximated by the discrete ones. 
Given two nonempty sets $\mathcal{B}, \mathcal{C} \in 2^{X}$, we write

$$
\mathcal{B}-\mathcal{C}=\{b-c: b \in \mathcal{B}, c \in \mathcal{C}\} \quad \text { and } \quad\|\mathcal{B}\|=\sup _{b \in \mathcal{B}}\|b\|
$$

The following theorem is a generalization to m-semigroups of a result proven in [27].

Theorem 2.6. Let $S(t)$ be a closed m-semigroup, possessing the global attractor $\mathcal{A}$, and let $\left\{S_{k}, 0<k \leq \kappa_{0}\right\}_{n \in \mathbb{N}}$ be a family of discrete closed m-semigroups, with global attractor $\mathcal{A}_{k}$. Assume the following:

(H1) there exists $\kappa_{1} \in\left(0, \kappa_{0}\right]$ such that the set

$$
\mathcal{K}=\bigcup_{k \in\left(0, \kappa_{1}\right]} \mathcal{A}_{k}
$$

is bounded in $X$;

(H2) there exists $t_{0} \geq 0$ such that for any $T^{\star}>t_{0}$,

$$
\lim _{k \rightarrow 0} \sup _{x \in \mathcal{A}_{k}, n k \in\left[t_{0}, T^{\star}\right]}\left\|S_{k}^{n} x-S(n k) x\right\|=0 .
$$

Then,

$$
\lim _{k \rightarrow 0} \operatorname{dist}\left(\mathcal{A}_{k}, \mathcal{A}\right)=0
$$

Proof. Let $\varepsilon>0$ and $k \in\left(0, \kappa_{1}\right]$. Since $\mathcal{K}$ is bounded and $\mathcal{A}$ is attracting, there exists $t_{\varepsilon}>t_{0} \geq 0$ such that

$$
\operatorname{dist}(S(t) \mathcal{K}, \mathcal{A})<\frac{\varepsilon}{2}, \quad \forall t \geq t_{\varepsilon}
$$

Let now $x_{k} \in \mathcal{A}_{k}$, and pick

$$
n_{k}=\left\lfloor\frac{t_{\varepsilon}+1}{k}\right\rfloor
$$

Thanks to the invariance of $\mathcal{A}_{k}$ under $S_{k}$, there exists $y_{k} \in \mathcal{A}_{k}$ such that $x_{k} \in S_{k}^{n_{k}} y_{k}$, and by (H2).

$$
\left\|x_{k}-S\left(n_{k} k\right) y_{k}\right\| \leq\left\|S_{k}^{n_{k}} y_{k}-S\left(n_{k} k\right) y_{k}\right\|<\frac{\varepsilon}{2}, \quad \forall k \leq \kappa_{\varepsilon},
$$

with a proper choice of $\kappa_{\varepsilon}>0$. As a consequence, if $y \in S\left(n_{k} k\right) y_{k}$,

$$
\begin{aligned}
\operatorname{dist}\left(\mathcal{A}_{k}, \mathcal{A}\right) & =\sup _{x_{k} \in \mathcal{A}_{k}} \inf _{x \in \mathcal{A}}\left\|x_{k}-x\right\| \leq \sup _{x_{k} \in \mathcal{A}_{k}}\left[\left\|x_{k}-y\right\|+\operatorname{dist}(y, \mathcal{A})\right] \\
& \leq \sup _{x_{k} \in \mathcal{A}_{k}}\left[\left\|x_{k}-S\left(n_{k} k\right) y_{k}\right\|+\operatorname{dist}\left(S\left(n_{k} k\right) y_{k}, \mathcal{A}\right)\right] \\
& \leq \sup _{x_{k} \in \mathcal{A}_{k}}\left\|x_{k}-S\left(n_{k} k\right) y_{k}\right\|+\operatorname{dist}\left(S\left(n_{k} k\right) \mathcal{K}, \mathcal{A}\right)<\varepsilon
\end{aligned}
$$

and the theorem is proved. 


\section{Applications to the Navier-Stokes Equations}

In this section, we apply the above abstract framework to the fully implicit Euler approximation of the Navier-Stokes equations. It is known that such a scheme does not generate a single-valued discrete semigroup, since uniqueness of solutions holds under a restriction on the time-step parameter which depends on the initial datum. To circumvent this difficulty, we will show that such a scheme generates a family of closed discrete m-semigroups depending on the time-step parameter, whose related attractors converge to the attractor of the dynamical system generated by the Navier-Stokes equations.

3.1. Function spaces. Let $\Omega \subset \mathbb{R}^{2}$ be a bounded domain with smooth boundary $\partial \Omega$. For $p \in[1, \infty]$ and $k \in \mathbb{N}$, we denote by $\mathbf{L}^{p}(\Omega)=\left\{L^{p}(\Omega)\right\}^{2}, \mathbf{H}^{k}(\Omega)=\left\{H^{k}(\Omega)\right\}^{2}$, and $\mathbf{H}_{0}^{k}(\Omega)=\left\{H_{0}^{k}(\Omega)\right\}^{2}$ the usual Lebesgue and Sobolev spaces of vector-valued functions on $\Omega$. Setting

$$
\mathcal{D}=\left\{u \in C_{0}^{\infty}\left(\Omega, \mathbb{R}^{2}\right): \operatorname{div} u=0\right\},
$$

we consider the usual Hilbert spaces associated with the Navier-Stokes equations

$$
\begin{aligned}
& H=\text { closure of } \mathcal{D} \text { in } \mathbf{L}^{2}(\Omega), \\
& V=\text { closure of } \mathcal{D} \text { in } \mathbf{H}^{1}(\Omega),
\end{aligned}
$$

where we denote by $|\cdot|,(\cdot, \cdot)$ and $\|\cdot\|,((\cdot, \cdot))$ the norm and the scalar product in $H$ and in $V$, respectively. Also, we indicate by $V^{*}$ the dual space of $V$, endowed with the usual dual norm $\|\cdot\|_{*}$, and by $\langle\cdot, \cdot\rangle$ the duality pairing between $V$ and $V^{*}$. Calling

$$
P: \mathbf{L}^{2}(\Omega)=H \oplus H^{\perp} \rightarrow H
$$

the Leray orthogonal projection, the Stokes operator is defined as

$$
A=-P \Delta, \quad \operatorname{dom}(A)=\mathbf{H}^{2}(\Omega) \cap V .
$$

It is well known that the operator $A$ is self-adjoint and strictly positive. Moreover, $\operatorname{dom}\left(A^{1 / 2}\right)=V$ and

$$
\|u\|=|\nabla u|=\left|A^{1 / 2} u\right|, \quad \forall u \in V .
$$

Setting

$$
B(u, v)=P[(u \cdot \nabla) v]
$$

system (1.1)-(1.3) can be rewritten as an abstract evolution equation of the form

$$
\left\{\begin{array}{l}
\dot{u}+\nu A u+B(u, u)=f, \\
u(0)=u_{0}
\end{array}\right.
$$

where $f=P f$, since we are assuming incompressible forcing. As proved in [23], problem (3.1) generates a continuous and dissipative single-valued dynamical system $S(t): H \rightarrow$ $H$, which possesses the global attractor $\mathcal{A}$, bounded in $V$. 
3.2. The implicit Euler scheme. A possible time discretization of (3.1) is given by the fully implicit Euler scheme

$$
\frac{u^{n}-u^{n-1}}{k}+\nu A u^{n}+B\left(u^{n}, u^{n}\right)=f, \quad u^{0}=u_{0},
$$

where $n \geq 1$ and $k>0$ is the time-step. Our goal in this section is to prove that (3.2) generates a closed discrete m-semigroup $\left\{S_{k}\right\}_{n \in \mathbb{N}}$. Indeed, as in the stationary NavierStokes problem, a solution to (3.2) is not in general unique. In particular, uniqueness of solutions may be proved requiring $k$ to be bounded from above by a constant depending on the initial datum. More specifically, such a bound vanishes as the norm of $u_{0}$ tends to infinity, and this makes it impossible to properly define a single-valued semigroup acting on the whole phase space. Nonetheless, by means of the tools devised in Section 2, we will still be able to give a description of the longtime behavior of the discretized Navier-Stokes equations, and discuss its convergence to the time-continuous asymptotic dynamics.

Notation. Throughout the section, $C$ and $\mathcal{Q}(\cdot)$ will denote a generic positive constant and a generic increasing positive function, respectively, whose value may change even in the same line of a certain equation. Unless otherwise stated, these quantities will be independent of $k, n$ and of the initial datum $u_{0}$. In general, they might depend on the structural quantities of the system $(\nu, f, \Omega)$.

Fix $k>0$. For $w \in H$, we look at the problem

$$
u+k \nu A u+k B(u, u)=w+k f
$$

for which we seek solutions in the following weak sense.

Definition 3.1. A vector $u \in V$ is a solution to (3.3) if

$$
(u, v)+k \nu((u, v))+k b(u, u, v)=(w, v)+k(f, v), \quad \forall v \in V,
$$

where $b(u, v, w)=\langle B(u, v), w\rangle$ is the usual trilinear form associated to the weak formulation of the Navier-Stokes equations.

Remark 3.2. Recall that the trilinear form $b$ satisfies the following properties:

$$
\begin{aligned}
& |b(u, v, w)| \leq C|u|^{1 / 2}|A u|^{1 / 2}\|v\||w|, \quad \forall u \in \operatorname{dom}(A), v \in V, w \in H, \\
& |b(u, v, w)| \leq C|u|^{1 / 2}\|u\|^{1 / 2}\|v\||w|^{1 / 2}\|w\|^{1 / 2}, \quad \forall u, v, w \in V, \\
& b(u, v, v)=0, \quad \forall u, v \in V .
\end{aligned}
$$

It is a classical result that a (possibly not unique) solution to (3.3) exists. Moreover, any solution $u \in V$ satisfies the energy estimate

$$
|u|^{2}+k \nu\|u\|^{2} \leq|w|^{2}+C k|f|^{2},
$$

where $C>0$ does not depend on $k$. For every $w \in H$, define the multivalued map $S_{k}: 2^{H} \rightarrow 2^{H}$ by

$$
S_{k} w=\{u \in V: u \text { solves (3.3) with time-step } k\} .
$$

Notice that, in light of (3.8), the set $S_{k} w$ is bounded in $V$ and therefore relatively compact in $H$, thanks to the compactness of the embedding $V \hookrightarrow H$. 
3.3. The discrete m-semigroup. Let us consider the discrete m-semigroup $\left\{S_{k}\right\}_{n \in \mathbb{N}}$ generated by $S_{k}$. It is now clear that, for every $n \in \mathbb{N}$,

$$
S_{k} u^{n-1}=\left\{u^{n} \in H: u^{n} \text { solves (3.2) with time-step } k\right\} .
$$

Also, a vector $u^{n} \in S_{k}^{n} u_{0}$ if and only if there exists a sequence of elements $\left(u^{0}, u^{1}, \ldots, u^{n-1}, u^{n}\right)$ such that $u^{i} \in S_{k} u^{i-1}$ for every $i=1, \ldots, n$ and $u^{0}=u_{0}$. From (3.8), we infer that any $u^{i} \in S_{k} u^{i-1}$ satisfies the energy estimate

$$
\left|u^{i}\right|^{2}+k \nu\left\|u^{i}\right\|^{2} \leq\left|u^{i-1}\right|^{2}+C k|f|^{2},
$$

and, inductively, any $u^{n} \in S_{k}^{n} u^{0}$ fulfills the bound

$$
\left|u^{n}\right|^{2}+k \nu \sum_{i=1}^{n}\left\|u^{i}\right\|^{2} \leq\left|u^{0}\right|^{2}+C k n|f|^{2} .
$$

We then have the following theorem.

Theorem 3.3. The multivalued map $S_{k}$ associated to the implicit Euler scheme (3.2) generates a closed discrete m-semigroup $\left\{S_{k}\right\}_{n \in \mathbb{N}}$.

Proof. Since properties (S.1) $($ S.2) are satisfied by definition, all we need to prove is that $S_{k}^{n}$ is a closed multivalued map for each $n \in \mathbb{N}$. As $j \rightarrow \infty$, let $u_{j}^{0} \rightarrow u^{0}$ in $H$ and $u_{j}^{n} \in S_{k}^{n} u_{j}^{0}$ with $u_{j}^{n} \rightarrow u^{n}$ in $H$. We have to show that $u^{n} \in S_{k}^{n} u^{0}$.

Since $u_{j}^{n} \in S_{k}^{n} u_{j}^{0}$, there exists a sequence $\left(u_{j}^{0}, u_{j}^{1}, \ldots, u_{j}^{n-1}, u_{j}^{n}\right)$ where $u_{j}^{i} \in S_{k} u_{j}^{i-1}$ is a solution to

$$
\left(u_{j}^{i}, v\right)+k \nu\left(\left(u_{j}^{i}, v\right)\right)+k b\left(u_{j}^{i}, u_{j}^{i}, v\right)=k(f, v)+\left(u_{j}^{i-1}, v\right), \quad \forall v \in V .
$$

Also, the fact that $u_{j}^{0} \rightarrow u^{0}$ in $H$ implies the existence of a positive number $M$ such that

$$
\sup _{j}\left|u_{j}^{0}\right|^{2} \leq M
$$

In view of (3.9), we obtain the bound

$$
\left|u_{j}^{i}\right|^{2}+k \nu \sum_{\ell=1}^{i}\left\|u_{j}^{\ell}\right\|^{2} \leq C k i|f|^{2}+M .
$$

Thus, for every $i=1, \ldots, n$, we have the following convergences (up to not relabeled subsequences) as $j \rightarrow \infty$ :

$$
u_{j}^{i} \rightarrow u^{i} \text {, strongly in } H \text { and weakly in } V \text {. }
$$

Now, passing to the limit in (3.10), we readily get that

$$
\left(u^{i}, v\right)+k \nu\left(\left(u^{i}, v\right)\right)+k b\left(u^{i}, u^{i}, v\right)=k(f, v)+\left(u^{i-1}, v\right), \quad \forall v \in V .
$$

As a consequence, $u^{i} \in S_{k} u^{i-1}$ for each $i=1, \ldots, n$. But then, $u^{n} \in S_{k} u^{n-1} \subset S_{k}^{n} u^{0}$, so $S_{k}^{n}$ is a closed map for every $n \in \mathbb{N}$.

From the energy estimate (3.9) and the fact that a closed map has necessarily closed values, we have the following straightforward consequence.

Corollary 3.4. The discrete m-semigroup $\left\{S_{k}\right\}_{n \in \mathbb{N}}$ has compact values, namely, the set $S_{k}^{n} u_{0}$ is compact in $H$ for every $u_{0} \in H$. 
3.4. Earlier Contributions. To continue our study, we first collect some results obtained in [25]. The first two concern initial data $u_{0} \in H$. There exists $\kappa_{0}>0$, independent of $u_{0}, n, k$, such that the following hold:

(D.1) For every $k>0$,

$$
\left|S_{k}^{n} u_{0}\right|^{2} \leq(1+k)^{-n}\left|u_{0}\right|^{2}+C|f|^{2}, \quad \forall n \geq 0
$$

(D.2) Let $k \in\left(0, \kappa_{0}\right]$. There exists a constant $R_{0}>0$ with the following property: for every $R \geq 0$, there exists $t_{0}=t_{0}(R) \geq 0$ such that

$$
\left|S_{k}^{n} u_{0}\right| \leq R_{0}, \quad \forall n k \geq t_{0},
$$

whenever $\left|u_{0}\right| \leq R$. Both $R_{0}$ and $t_{0}$ can be explicitly computed and do not depend on $n$ and $k$. In other words, the set

$$
\mathcal{B}_{0}=\left\{v \in H:|v| \leq R_{0}\right\}
$$

is a bounded absorbing set for $\left\{S_{k}\right\}_{n \in \mathbb{N}}$.

We now turn our attention to initial data $u_{0} \in V$ and recall the main result derived in [25], tailored to our case.

Theorem 3.5. Suppose $\left\|u_{0}\right\| \leq R$, and let the time-step $k$ be such that

$$
k \leq \kappa_{\star}(R)=\min \left\{\kappa_{0}, \frac{1}{\mathcal{Q}(R)}, C\right\} .
$$

Then the estimate

$$
\left\|S_{k}^{n} u_{0}\right\| \leq \mathcal{Q}(R)
$$

holds true for every $n \geq 1$.

Remark 3.6. Since the issue of non-uniqueness of solutions to (3.3) is the main motivation for this work, let us briefly discuss one way to recover uniqueness of solution to (3.2), in the sense of (3.4) with $u=u^{n}$ and $w=u^{n-1}$. Let $u_{1}^{n}$ and $u_{2}^{n}$ be two solutions corresponding to the same initial data $u_{0} \in V$, let $R \geq 0$ be such that $\left\|u_{0}\right\| \leq R$, and set $u^{n}=u_{1}^{n}-u_{2}^{n}$. If $k \leq \kappa_{\star}(R)$, from (3.4) and (3.7) we learn that

$$
\left|u^{n}\right|^{2}+k \nu\left\|u^{n}\right\|^{2}=-k b\left(u^{n}, u_{2}^{n}, u^{n}\right) .
$$

Now, using (3.6) and Theorem 3.5, for any $n \geq 1$ we obtain

$$
\begin{aligned}
\left|u^{n}\right|^{2}+k \nu\left\|u^{n}\right\|^{2} & \leq C k\left|u^{n}\right|\left\|u^{n}\right\|\left\|u_{2}^{n}\right\| \leq \mathcal{Q}(R) k\left|u^{n}\right|\left\|u^{n}\right\| \\
& \leq \frac{1}{2}\left|u^{n}\right|^{2}+\mathcal{Q}(R)^{2} k^{2}\left\|u^{n}\right\|^{2} .
\end{aligned}
$$

Therefore, if we require

$$
k \leq \min \left\{\kappa_{\star}(R), \frac{\nu}{2 \mathcal{Q}(R)^{2}}\right\}
$$

we can conclude that

$$
\left|u^{n}\right|^{2}+k \nu\left\|u^{n}\right\|^{2} \leq 0 .
$$


This estimate clearly yields uniqueness of solutions. However, two main drawbacks arise. Firstly, we necessarily need $u_{0} \in V$, which rules out the possibility of defining a singlevalued semigroup on the natural phase space $H$ of weak solutions. Secondly, the restriction on $k$ depends on $\left\|u_{0}\right\|$, and thus uniqueness of solutions depends, in the end, on the single trajectory chosen and not uniformly with respect to the initial datum. This is why we decided to tackle this problem exploiting the machinery of multivalued semigroups.

3.5. The discrete global attractors. The higher order estimate in (3.12) is not enough to conclude the existence of the global attractor for the discrete m-semigroup $S_{k}$, since it requires the initial data $u_{0}$ to be in the more regular space $V$. Moreover, (3.11) shows a dependence of $\kappa_{\star}$ on the initial data, which turns out to be unsatisfactory in the approximation process devised in Theorem 2.6. We now show how to overcome this difficulty. First of all, thanks to the existence of a bounded absorbing set, it is natural to consider only initial data $u_{0} \in \mathcal{B}_{0}$.

Lemma 3.7. Let $k \in\left(0, \kappa_{0}\right]$, and consider a sequence $\left(u^{0}, u^{1}, \ldots\right)$, where $u^{0}=u_{0} \in \mathcal{B}_{0}$ and $u^{i} \in S_{k}^{i} u^{0}$. Then, there exists $\ell_{k} \in \mathbb{N}$ such that

$$
\left\|u^{\ell_{k}}\right\| \leq R_{\star}
$$

where $R_{\star}>0$ does not depend on $n, k$ and $u_{0}$.

Proof. Let

$$
n_{k}=\left\lfloor\frac{1}{k}\right\rfloor+1
$$

Estimate (3.9) immediately implies

$$
k \nu \sum_{i=1}^{n_{k}}\left\|u^{i}\right\|^{2} \leq R_{0}^{2}+C k n_{k}|f|^{2} .
$$

Arguing by contradiction, we infer that there exists $\ell_{k} \in\left\{1, \ldots, n_{k}\right\}$ such that

$$
k n_{k} \nu\left\|u^{\ell_{k}}\right\|^{2} \leq R_{0}^{2}+C k n_{k}|f|^{2} .
$$

Hence,

$$
\left\|u^{\ell_{k}}\right\|^{2} \leq \frac{R_{0}^{2}}{k n_{k} \nu}+C|f|^{2}
$$

Since $k n_{k} \geq 1$, the proof ends by setting

$$
R_{\star}^{2}=\frac{R_{0}^{2}}{\nu}+C|f|^{2} .
$$

Having in mind condition (3.11), we now fix $\kappa_{1}=\kappa_{\star}\left(R_{\star}\right) \leq \kappa_{0}$. Combining together (3.12) and the above Lemma 3.7 we obtain the following.

Corollary 3.8. Let $k \in\left(0, \kappa_{1}\right]$, and consider a sequence $\left(u^{0}, u^{1}, \ldots\right)$, where $u^{0}=u_{0} \in \mathcal{B}_{0}$ and $u^{i} \in S_{k}^{i} u^{0}$. Then, there exists $\ell_{k} \in \mathbb{N}$ such that

$$
\left\|u^{\ell_{k}+n}\right\| \leq R_{1}, \quad \forall n \geq 1
$$


As a consequence, for every $k \in\left(0, \kappa_{1}\right]$, there exists $n_{k} \in \mathbb{N}$ such that

$$
\left\|S_{k}^{n_{k}+n} u_{0}\right\| \leq R_{1}, \quad \forall n \geq 1 .
$$

Proof. The constant $R_{1}=\mathcal{Q}\left(R_{\star}\right)$ is given by (3.12), and it is clearly independent of $n, k$ and $u_{0}$. In the first estimate in the statement of the corollary, $\ell_{k}$ might depend on the particular sequence $\left(u^{0}, u^{1}, \ldots\right)$ which originates from $u^{0}=u_{0}$. As in the proof of Lemma 3.7, the choice

$$
n_{k}=\left\lfloor\frac{1}{k}\right\rfloor+1
$$

together with (3.12) and the fact that $\ell_{k} \leq n_{k}$, takes away this dependence and allows to obtain the uniform estimate (3.15).

Notice that (3.15) can be rewritten in the equivalent way

$$
\left\|S_{k}^{n} u_{0}\right\| \leq R_{1}, \quad \forall n \geq n_{k}+1
$$

and from the definition of $n_{k}$, we have that, in particular,

$$
\left\|S_{k}^{n} u_{0}\right\| \leq R_{1}, \quad \forall n k \geq 1+2 \kappa_{1} .
$$

We summarize the above observations in the next theorem, which, in fact, improves the results in 25 .

Theorem 3.9. Let $\kappa_{1}>0$ as above and $k \in\left(0, \kappa_{1}\right]$. There exists a constant $R_{1}>0$ with the following property: for every $R \geq 0$, there exists $t_{1}=t_{1}(R) \geq 0$ such that

$$
\left\|S_{k}^{n} u_{0}\right\| \leq R_{1}, \quad \forall n k \geq t_{1},
$$

whenever $\left|u_{0}\right| \leq R$. Both $R_{1}$ and $t_{1}$ can be explicitly computed and do not depend on $n$ and $k$. Hence, the set

$$
\mathcal{B}_{1}=\left\{v \in V:\|v\| \leq R_{1}\right\}
$$

is a $V$-bounded absorbing set for $\left\{S_{k}\right\}_{n \in \mathbb{N}}$.

Proof. Fix $k \in\left(0, \kappa_{1}\right]$ and let $\left|u_{0}\right| \leq R$. From (D.2), there exists $t_{0}=t_{0}(R)$ such that

$$
S_{k}^{n} u_{0} \in \mathcal{B}_{0}, \quad \forall n k \geq t_{0} .
$$

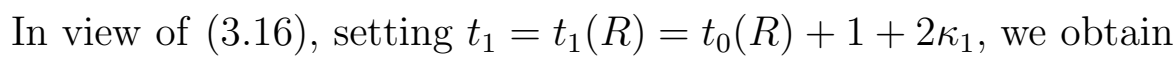

$$
S_{k}^{n} u_{0} \in \mathcal{B}_{1}, \quad \forall n k \geq t_{1},
$$

concluding the proof.

Remark 3.10. The result contained in the above Theorem 3.9 improves the one in [25] in two directions. On one hand, we only require the initial data to be in $H$. This shows a regularization property analogous to the one enjoyed by the solution to the NavierStokes equations. On the other hand, we obtain a uniform restriction on the time-step $k$, independent of the initial data.

Thanks to the above results, the discrete m-semigroup $\left\{S_{k}\right\}_{n \in \mathbb{N}}$ satisfies the assumptions of Theorem 2.4.

Proposition 3.11. For every $k \in\left(0, \kappa_{1}\right]$, there exists the global attractor $\mathcal{A}_{k}$ of the $m$ semigroup $\left\{S_{k}\right\}_{n \in \mathbb{N}}$. 
Remark 3.12. The global attractor $\mathcal{A}_{k}$ being the smallest closed attracting set of the phase space, from (3.17) we obtain the inclusion

$$
\mathcal{A}_{k} \subset \mathcal{B}_{1}
$$

The fact that $R_{1}$ does not depend on $k$ then yields

$$
\bigcup_{k \in\left(0, \kappa_{1}\right]} \mathcal{A}_{k} \subset \mathcal{B}_{1}
$$

Hence, the attractors $\mathcal{A}_{k}$ enjoy a uniform regularity property.

3.6. The attractor approximation. In this paragraph, we prove that the longterm behavior of the semigroup $S(t)$ generated by the Navier-Stokes equations (3.1) is approximated, in the sense of Theorem 2.6, by that of the discrete m-semigroup related to the fully implicit Euler scheme (3.2). The main result of this section reads as follows.

Theorem 3.13. The family of attractors $\left\{\mathcal{A}_{k}\right\}_{k \in\left(0, \kappa_{1}\right]}$ converges, as $k \rightarrow 0$, to $\mathcal{A}$, namely,

$$
\lim _{k \rightarrow 0} \operatorname{dist}\left(\mathcal{A}_{k}, \mathcal{A}\right)=0,
$$

where dist denotes the Hausdorff semidistance in $H$.

Our goal is to apply Theorem 2.6 to obtain the convergence of the discrete attractors $\mathcal{A}_{k}$ to the continuous time attractor $\mathcal{A}$ of the semigroup $S(t)$. By virtue of (3.19), assumption (H1) is automatically satisfied. The remaining of the section is devoted to the verification of the uniform convergence required by (H2). Since (H2) involves an estimate in terms of initial data belonging to $\mathcal{A}_{k}$, till the end of the section we will assume

$$
u_{0} \in \mathcal{B}_{1} \text {. }
$$

Define $\kappa_{2}=\min \left\{\kappa_{1}, \kappa_{\star}\left(R_{1}\right)\right\}$, where $\kappa_{\star}$ is given by (3.11). Notice that since $\mathcal{B}_{1}$ is absorbing, in view of Theorem 3.5 we have the following uniform estimate

$$
\sup _{k \in\left(0, \kappa_{2}\right]} \sup _{n \geq 0}\left\|S_{k}^{n} u_{0}\right\| \leq C \text {. }
$$

which, in turn, implies

$$
\sum_{n=i}^{m}\left\|u^{n}-u^{n-1}\right\|^{2} \leq C k(m-i+1)+C, \quad \forall i=1, \ldots, m,
$$

where $u^{n} \in S_{k} u^{n-1}$ for every $n=i, \ldots, m$. Indeed, multiplying (3.2) by $2 k A u^{n}$, we obtain

$$
\begin{aligned}
\left\|u^{n}\right\|^{2}-\left\|u^{n-1}\right\|^{2}+\left\|u^{n}-u^{n-1}\right\|^{2} & +2 \nu k\left|A u^{n}\right|^{2} \\
& +2 k b\left(u^{n}, u^{n}, A u^{n}\right)=2 k\left(f, A u^{n}\right) .
\end{aligned}
$$

Estimating the trilinear form using (3.5) and the bound (3.21), we infer that

$$
2 k b\left(u^{n}, u^{n}, A u^{n}\right) \leq 2 C k\left|u^{n}\right|^{1 / 2}\left\|u^{n}\right\|\left|A u^{n}\right|^{3 / 2} \leq \frac{\nu k}{2}\left|A u^{n}\right|^{2}+C k .
$$

Also,

$$
2 k\left(f, A u^{n}\right) \leq 2 k|f|\left|A u^{n}\right| \leq \frac{\nu k}{2}\left|A u^{n}\right|^{2}+C k
$$


Thus,

$$
\left\|u^{n}\right\|^{2}-\left\|u^{n-1}\right\|^{2}+\left\|u^{n}-u^{n-1}\right\|^{2}+\nu k\left|A u^{n}\right|^{2} \leq C k .
$$

Now, summing over $n=i, \ldots, m$ and neglecting the positive term $\left|A u^{n}\right|^{2}$, we get

$$
\left\|u^{m}\right\|^{2}-\left\|u^{i-1}\right\|^{2}+\sum_{n=i}^{m}\left\|u^{n}-u^{n-1}\right\|^{2} \leq C k(m-i+1) .
$$

Hence, a further application of (3.21) entails (3.22).

For any $k>0$, we define the piecewise constant and the piecewise linear functions

$$
u_{k}(t)=u^{n}, \quad t \in[(n-1) k, n k)
$$

and

$$
\tilde{u}_{k}(t)=u^{n}+\frac{t-n k}{k}\left(u^{n}-u^{n-1}\right), \quad t \in[(n-1) k, n k) .
$$

Notice that $\tilde{u}_{k}(n k)=u^{n}$. Also, it is easily seen that $\tilde{u}_{k}$ solves

$$
\dot{\tilde{u}}_{k}+\nu A \tilde{u}_{k}+B\left(\tilde{u}_{k}, \tilde{u}_{k}\right)=f+\Psi_{k}
$$

where

$$
\Psi_{k}(t)=\nu A\left(\tilde{u}_{k}(t)-u_{k}(t)\right)+B\left(\tilde{u}_{k}(t), \tilde{u}_{k}(t)\right)-B\left(u_{k}(t), u_{k}(t)\right) .
$$

Lemma 3.14. For any $T^{\star}>0, \Psi_{k} \in L^{2}\left(0, T^{\star} ; V^{*}\right)$ and

$$
\left\|\Psi_{k}\right\|_{L^{2}\left(0, T^{\star} ; V^{*}\right)}^{2} \leq k \mathcal{Q}\left(T^{\star}\right) .
$$

Proof. Let $v \in V$ be such that $\|v\| \leq 1$, and let $t \in[(n-1) k, n k)$ be fixed. In light of (3.6), we have

$$
\begin{aligned}
\left|\left\langle B\left(\tilde{u}_{k}, \tilde{u}_{k}\right)-B\left(u_{k}, u_{k}\right), v\right\rangle\right| & =\left|b\left(\tilde{u}_{k}, \tilde{u}_{k}-u_{k}, v\right)+b\left(\tilde{u}_{k}-u_{k}, u_{k}, v\right)\right| \\
& \leq C\left(\left\|\tilde{u}_{k}\right\|+\left\|u_{k}\right\|\right)\left\|\tilde{u}_{k}-u_{k}\right\| .
\end{aligned}
$$

Since the uniform bound (3.21) implies

$$
\left\|u_{k}(t)\right\|=\left\|u^{n}\right\| \leq C
$$

and

$$
\left\|\tilde{u}_{k}(t)\right\| \leq\left\|u^{n}\right\|+\left|\frac{t-n k}{k}\right|\left(\left\|u^{n}\right\|+\left\|u^{n-1}\right\|\right) \leq C,
$$

we infer that

$$
\left|\left\langle B\left(\tilde{u}_{k}, \tilde{u}_{k}\right)-B\left(u_{k}, u_{k}\right), v\right\rangle\right| \leq C\left\|\tilde{u}_{k}-u_{k}\right\| \leq C\left\|u^{n}-u^{n-1}\right\| .
$$

Clearly,

$$
\left|\left\langle A\left(\tilde{u}_{k}-u_{k}\right), v\right\rangle\right| \leq\left\|\tilde{u}_{k}-u_{k}\right\| \leq C\left\|u^{n}-u^{n-1}\right\| .
$$

Hence, for $t \in[(n-1) k, n k)$, we can conclude that

$$
\left\|\Psi_{k}(t)\right\|_{*} \leq C\left\|u^{n}-u^{n-1}\right\| .
$$


Thus, setting $N^{\star}=\left\lfloor T^{\star} / k\right\rfloor$, by the above bound and (3.22) we finally get

$$
\begin{aligned}
\left\|\Psi_{k}\right\|_{L^{2}\left(0, T^{\star} ; V^{*}\right)}^{2} & =\int_{0}^{T^{\star}}\left\|\Psi_{k}(t)\right\|_{*}^{2} \mathrm{~d} t \leq \sum_{n=1}^{N^{\star}+1} \int_{(n-1) k}^{n k}\left\|\Psi_{k}(t)\right\|_{*}^{2} \mathrm{~d} t \\
& \leq C k \sum_{n=1}^{N^{\star}+1}\left\|u^{n}-u^{n-1}\right\|^{2} \leq k \mathcal{Q}\left(T^{\star}\right) .
\end{aligned}
$$

Thus, the lemma is proved.

We are now ready to verify (a slightly stronger version of) assumption (H2) for our discrete m-semigroup, which will conclude the proof of Theorem 3.13 ,

Lemma 3.15. For any $T^{\star}>0$,

$$
\lim _{k \rightarrow 0} \sup _{u_{0} \in \mathcal{B}_{1}, n k \in\left[0, T^{\star}\right]}\left|S_{k}^{n} u_{0}-S(n k) u_{0}\right|=0 .
$$

Proof. Let $u=u(t)=S(t) u_{0}$ be the solution to (3.1). As shown in [23], $S(t)$ satisfies the uniform energy estimate

$$
\sup _{t \geq 0} \sup _{u_{0} \in \mathcal{B}_{1}}\left\|S(t) u_{0}\right\| \leq C .
$$

For $\tilde{u}_{k}$ defined as above and $k \in\left(0, \kappa_{2}\right]$, consider the difference $v_{k}=u-\tilde{u}_{k}$, which is a solution to

$$
\dot{v}_{k}+\nu A v_{k}+B\left(v_{k}, u\right)+B\left(\tilde{u}_{k}, v_{k}\right)=-\Psi_{k} .
$$

Testing the above equation by $v_{k}$, one obtains

$$
\frac{1}{2} \frac{\mathrm{d}}{\mathrm{d} t}\left|v_{k}\right|^{2}+\nu\left\|v_{k}\right\|^{2}+b\left(v_{k}, u, v_{k}\right)=-\left\langle\Psi_{k}, v_{k}\right\rangle .
$$

By (3.6) and (3.26), the trilinear form can be estimated as

$$
\left|b\left(v_{k}, u, v_{k}\right)\right| \leq C\left|v_{k}\right|\left\|v_{k}\right\|\|u\| \leq \frac{\nu}{4}\left\|v_{k}\right\|^{2}+C\left|v_{k}\right|^{2},
$$

and from the obvious bound

$$
-\left\langle\Psi_{k}, v_{k}\right\rangle \leq \frac{\nu}{4}\left\|v_{k}\right\|^{2}+C\left\|\Psi_{k}\right\|_{*}^{2}
$$

we derive the differential inequality

$$
\frac{\mathrm{d}}{\mathrm{d} t}\left|v_{k}\right|^{2} \leq C\left|v_{k}\right|^{2}+C\left\|\Psi_{k}\right\|_{*}^{2}
$$

Since $v_{k}(0)=0$, an application of the Gronwall inequality together with Lemma 3.14 gives

$$
\left|v_{k}(t)\right|^{2} \leq C \mathrm{e}^{C t} \int_{0}^{t}\left\|\Psi_{k}(s)\right\|_{*}^{2} \mathrm{~d} s \leq C \mathrm{e}^{C T^{\star}}\left\|\Psi_{k}\right\|_{L^{2}\left(0, T^{\star} ; V^{*}\right)}^{2} \leq k \mathcal{Q}\left(T^{\star}\right) .
$$

As a consequence,

$$
\left|u(t)-\tilde{u}_{k}(t)\right|^{2} \leq k \mathcal{Q}\left(T^{\star}\right)
$$


But then

$$
\begin{aligned}
\lim _{k \rightarrow 0} & \sup _{u_{0} \in \mathcal{B}_{1}, n k \in\left[0, T^{\star}\right]}\left|S_{k}^{n} u_{0}-S(n k) u_{0}\right| \\
= & \lim _{k \rightarrow 0} \sup _{u_{0} \in \mathcal{B}_{1}, n k \in\left[0, T^{\star}\right]} \sup _{u^{n} \in S_{k}^{n} u_{0}}\left|u^{n}-u(n k)\right| \\
= & \lim _{k \rightarrow 0} \sup _{u_{0} \in \mathcal{B}_{1}, n k \in\left[0, T^{\star}\right]} \sup _{u^{n} \in S_{k}^{n} u_{0}}\left|\tilde{u}_{k}(n k)-u(n k)\right|=0
\end{aligned}
$$

and the proof is over.

Acknowledgments. This work was partially supported by the National Science Foundation under the grant NSF-DMS-0906440, and by the Research Fund of Indiana University.

\section{REFERENCES}

[1] J-P. Aubin, H. Frankowska, Set Valued Analysis, Birkhäuser, Boston, 1990.

[2] J.M. Ball, Continuity properties and global attractors of generalized semiflows and the Navier-Stokes equations, J. Nonlinear Sci. 7 (1997), 475-502.

[3] V. Barbu, Nonlinear semigroups and differential equations in Banach spaces, Noordhoff International Publishing, Leiden, 1976.

[4] A.V. Babin, M.I. Vishik, Attractors of evolution equations, North-Holland, Amsterdam, 1992.

[5] T. Caraballo, J.A. Langa, V.S. Melnik, J. Valero, Pullback attractors of nonautonomous and stochastic multivalued dynamical systems, Set-Valued Anal. 2 (2003), 153-201.

[6] T. Caraballo, P. Marín-Rubio, J.C. Robinson, A comparison between two theories for multi-valued semiflows and their asymptotic behaviour, Set-Valued Anal. 11 (2003), 297-322.

[7] V.V. Chepyzhov, M.I. Vishik, Attractors for equations of mathematical physics, Amer. Math. Soc., Providence, 2002.

[8] C.B. Gentile, J. Simsen, On attractors for multivalued semigroups defined by generalized semiflows, Set-Valued Anal. 16 (2008), 105-124.

[9] J.K. Hale, Asymptotic behavior of dissipative systems, Amer. Math. Soc., Providence, 1988.

[10] J.K. Hale, X. Lin, G. Raugel, Upper semicontinuity of attractors for approximations of semigroups and partial differential equations, Math. Comp. 50 (1988), 89-123.

[11] E. Hill, A.T. Süli, Approximation of the global attractor for the incompressible Navier-Stokes equations, IMA J. Numer. Anal. 20 (2000), 633-667.

[12] N. Ju, On the global stability of a temporal discretization scheme for the Navier-Stokes equations, IMA J. Numer. Anal. 22 (2002), 577-597.

[13] A.V. Kapustian, J. Valero, Attractors of multivalued semiflows generated by differential inclusions and their approximations, Abstr. Appl. Anal. 5 (2000), 33-46.

[14] V.S. Melnik, J. Valero, On attractors of multivalued semi-flows and differential inclusion, Set-Valued Anal. 6 (1998), 83-111.

[15] V. Pata, S. Zelik, A result on the existence of global attractors for semigroups of closed operators, Commun. Pure Appl. Anal. 6 (2007), 481-486.

[16] J.C. Robinson, Infinite-dimensional dynamical systems, Cambridge University Press, Cambridge, 2001.

[17] R. Rossi, S. Segatti, U. Stefanelli, Attractors for gradient flows of nonconvex functionals and applications, Arch. Ration. Mech. Anal. 187 (2008), 91-135.

[18] G. Schimperna, S. Segatti, U. Stefanelli, Well-posedness and long-time behavior for a class of doubly nonlinear equations, Discrete Contin. Dyn. Syst. 18 (2007), 15-38.

[19] J. Shen, Convergence of approximate attractors for a fully discrete system for reaction-diffusion equations, Numer. Funct. Anal. and Optim. 10 (1989), 1213-1234.

[20] H. Sohr, The Navier-Stokes equations, Birkhäuser, Basel, 2001. 
[21] A.M. Stuart and A.R. Humphries, Dynamical systems and numerical analysis, Cambridge University Press, Cambridge, 1996.

[22] R. Temam, Navier-Stokes equations and nonlinear functional analysis, CBMS-NSF Regional conference Series in Applied Mathematics, SIAM, Philadelphia, 1983.

[23] R. Temam, Infinite-dimensional dynamical systems in mechanics and physics, Springer-Verlag, New York, 1997.

[24] R. Temam, Navier-Stokes equations, AMS Chelsea Publishing, Providence, 2001.

[25] F. Tone, D. Wirosoetisno, On the long-time stability of the implicit Euler scheme for the twodimensional Navier-Stokes equations, SIAM J. Numer. Anal. 44 (2006), 29-40.

[26] F. Tone, X. Wang, Approximation of the stationary statistical properties of the dynamical system generated by the two-dimensional Rayleigh-Benard convection problem, Anal. Appl. 9 (2011), 421446.

[27] X. Wang, Approximation of stationary statistical properties of dissipative dynamical systems: time discretization, Math. Comp. 79 (2010), 259-280.

Indiana University - Department of Mathematics

RaWles Hall, Bloomington, IN 47405, USA

E-mail address: micotize@indiana.edu (M. Coti Zelati)

University of West Florida - Department of Mathematics and Statistics

Pensacola, FL 32514, USA

E-mail address: ftone@uwf.edu (F. Tone) 